\title{
Leptophilic composite asymmetric dark matter and its detection
}

\author{
Mengchao Zhang $\odot^{*}$ \\ Department of Physics and Siyuan Laboratory, Jinan University, \\ Guangzhou 510632, People's Republic of China
}

(Received 11 June 2021; accepted 19 August 2021; published 8 September 2021)

\begin{abstract}
We propose a model which explains the baryon asymmetry of the universe and dark matter relic density at the same time. In this model, dark matter candidate is the dark baryon composed by dark quarks. A scalar mediator, which couples to the standard model leptons and dark quarks, is introduced to generate the asymmetry of baryon and dark baryon simultaneously. Direct detection and collider detection of this model are studied. We find that current underground direct detection experiments and LHC can hardly detect this model. But future lepton colliders, such as CEPC, have great potential to detect a large portion of the model parameter space, via novel signal "displaced lepton jet".
\end{abstract}

DOI: $10.1103 /$ PhysRevD.104.055008

\section{INTRODUCTION}

The observed baryon asymmetry of the universe (BAU) is a main puzzle in modern cosmology and particle physics. The ratio of the baryon number density to entropy density, $Y_{\Delta B}=\left(n_{B}-n_{\bar{B}}\right) / s \approx 8 \times 10^{-11}$, is measured by the cosmic microwave backgound (CMB) [1] and big bang nucleosynthesis (BBN) [2]. To explain BAU, three Sakharov conditions [3] need to be satisfied: baryon number violation, $C$ and $C P$ violation, and departure from thermal equilibrium. Successful baryogenesis mechanisms include electroweak baryogenesis [4-6], leptogenesis [7], or the Affleck-Dine mechanism [8,9]. Recent review see Ref. [10].

Another important issue in particle cosmology is the nature of dark matter (DM). So far, we only know the existence of DM through its gravitational effects [11]. The lack of observations other than the gravitational effects makes the DM model building highly speculative. Weakly interacting massive particle (WIMP) DM, which can naturally explain observed DM relic density by freezeout mechanism, has been intensively searched for by indirect detection (ID) experiments, direct detection (DD) experiments, and collider experiments [12-18]. But definite observation evidence of WIMP has not been seen in above searches.

Motivated by the null results in WIMP DM searches and the coincidence that the abundance of baryon and DM are

\footnotetext{
* Corresponding author. mczhang@jnu.edu.cn.

Published by the American Physical Society under the terms of the Creative Commons Attribution 4.0 International license. Further distribution of this work must maintain attribution to the author(s) and the published article's title, journal citation, and DOI. Funded by SCOAP ${ }^{3}$.
}

very close $\left(\Omega_{\mathrm{DM}} \simeq 5 \Omega_{\mathrm{B}}\right)$, it is natural to assume that the origin of baryon and DM abundance might be related to each other. To be more specific, the DM abundance is determined by the asymmetry between DM and anti-DM, and the asymmetry in dark sector and visible sector are from the same source. Such an asymmetric dark matter (ADM) paradigm is very different from WIMP in terms of model building and phenomenology study [19-45].

In this paper we focus on the composite ADM model $[38,39,42,43,46-49]$. In composite ADM model, there is a confined QCD-like strong interaction in the dark sector, and the DM candidate is the lightest dark baryon. Thus the mass of DM is basically determined by the dark confinement scale $\Lambda_{\mathrm{OCD}}^{\prime}{ }^{1}$. Furthermore, we use the decay of mediator particle (labeled as $\Phi$ ) to generate the asymmetry in both visible sector and dark sector, as proposed in [45]. The mediator $\Phi$ carries particle numbers in both sectors. Introducing such a "bi-charged" mediator have many advantages. First, the asymmetry in both visible and dark sector comes from the asymmetry of mediator $\Phi$, and thus the particle number densities in two sectors are automatically connected to each other. Second, this mediator provides a portal which release the entropy in the dark sector to visible sector, and thus prevent the dark sector from being hot $[38,44]$. Finally, as we will explain later on, the mass of mediator $\Phi$ can be quite low, and thus makes this model detectable at current or coming experiments.

In previous work [45], mediator $\Phi$ is chosen to couple to a dark quark (labeled as $q^{\prime}$ ) and a standard model (SM) quark $q$. Being charged under the SM QCD, $\Phi$ can be copiously produced at the LHC. Energetic dark quark $q^{\prime}$ in the final state, which comes from $\Phi$ decay $\Phi \rightarrow q^{\prime} \bar{q}$, will eventually evolves to a so-called "dark-jet." Such a novel

\footnotetext{
${ }^{1}$ In this paper we use ${ }^{\prime}$ to denote objects in the dark sector.
} 
collider signature has induced many studies in recent years [45,50-71]. In this paper we discuss another possibility for composite ADM model: mediator $\Phi$ couples to a dark quark and a lepton, instead of a dark quark and a SM quark. The rapid sphaleron process in the early universe can transfer the lepton asymmetry generated from $\Phi$ decay into baryon asymmetry, and thus BAU can also be explained in this model. Different with the model proposed in [45], the dark sector in this model mainly talks to the lepton sector in the SM. Such a leptophilic composite ADM model can be detected by direct detection (DD) experiments through the scattering with electrons. This model can also be detected at colliders by novel signatures like displaced lepton jets. We will choose LHC and projected CEPC [72] as representative detectors for collider searches.

In the next section, we will construct an ADM model which explain the BAU and DM relic density simultaneously. In Sec. III we discuss the constrains on this model from DD experiments. Section IV is dedicated to the collider searches. In Sec. V we conclude this work.

\section{THE GENERATION OF BARYON AND DARK BARYON ASYMMETRY}

The model we present in this section is similar to the model proposed in [45]. First, we need to generate the asymmetry of mediator $\Phi$. This goal can be achieved by the out-of-equilibrium and $C P$ violated decay of a heavy Majorana fermion, which is just like the leptogenesis process $[7,73,74]$. Furthermore, we need to break the conservation of dark baryon number (labeled as $B^{\prime}$ ) when the temperature of universe is high. In addition to the scalar mediator $\Phi$ and dark quark $q^{\prime},{ }^{2}$ we extend the SM by two heavy Majorana fermions $\left(N_{1}\right.$ and $\left.N_{2}\right)$ for out-of-equilibrium process, and a Dirac fermion $\chi$ charged under dark QCD (denoted as $S U(3)^{\prime}$ ) to break the dark baryon number $B^{\prime}$. In Table I we present charge, spin, and particle number carried by each particle.

We extend the SM Lagrangian with the particles present in Table I:

$$
\begin{aligned}
\mathcal{L}= & \mathcal{L}_{\mathrm{SM}}-\frac{1}{2} \sum_{i=1,2} M_{N_{i}} \bar{N}_{i} N_{i}^{C}-m_{\Phi}^{2} \Phi^{\dagger} \Phi-m_{\chi} \bar{\chi} \chi \\
& -m_{q^{\prime}} \bar{q}^{\prime} q^{\prime}+\mathcal{L}_{\text {kinetic }} \\
& -\sum_{i=1,2} \lambda_{i} \bar{N}_{i} \chi \Phi^{\dagger}-\kappa \Phi{\overline{q^{\prime}}}_{L} l_{R}-\frac{1}{\Lambda_{1}^{2}}\left(\bar{q}^{\prime C} \chi\right)\left(\bar{q}^{\prime}{ }_{L}^{C} l_{R}\right) \\
& -\frac{1}{\Lambda_{2}^{2}}\left(\bar{\chi} \gamma^{\mu} q^{\prime}\right)\left(\bar{d}_{R} \gamma_{\mu} u_{R}\right)+\text { H.c. }
\end{aligned}
$$

In the above Lagrangian, we dismiss the traditional leptogenesis operator $N_{i} L H$ by assuming this term to be

\footnotetext{
${ }^{2}$ We can certainly consider more than one flavor of dark quark, but the number of dark quark flavor is not relevant in this section.
}

TABLE I. Particles contents and their property. Here we present charges, spins, and particle numbers carried by each particles. $N_{1} / N_{2}$ are heavy Majorana fermions. $\Phi$ is the scalar mediator that carries the SM lepton number $L$ and dark baryon number $B^{\prime}$. $\chi$ is a Dirac fermion that carries the same particle number and charge as $\Phi$ carries. $q^{\prime}$ is the dark quark. $l$ denote the SM leptons $e, \mu$, and $\tau$. $d_{R}$ and $u_{R}$ are the SM right-handed down quark and up quark.

\begin{tabular}{lccccccc}
\hline \hline & $S U(3)^{\prime}$ & $S U(3)$ & $U_{Y}(1)$ & Spin & $L$ & $B$ & $B^{\prime}$ \\
\hline$N_{1} / N_{2}$ & 1 & 1 & 0 & $1 / 2$ & 0 & 0 & 0 \\
$\Phi$ & 3 & 1 & 1 & 0 & -1 & 0 & $1 / 3$ \\
$\chi$ & 3 & 1 & 1 & $1 / 2$ & -1 & 0 & $1 / 3$ \\
$q^{\prime}$ & 3 & 1 & 0 & $1 / 2$ & 0 & 0 & $1 / 3$ \\
$l_{R}$ & 1 & 1 & -1 & $1 / 2$ & 1 & 0 & 0 \\
$d_{R}$ & 1 & 3 & $-1 / 3$ & $1 / 2$ & 0 & $1 / 3$ & 0 \\
$u_{R}$ & 1 & 3 & $2 / 3$ & $1 / 2$ & 0 & $1 / 3$ & 0 \\
\hline \hline
\end{tabular}

negligible, because we do not want to mix the standard leptogenesis with our scenario. Furthermore, we introduce two dimension- 6 four fermions operators to make $\chi$ decay. ${ }^{3}$ Actually, due to angular momentum conservation and gauge invariance, we can not write down dimension-4 operator to make $\chi$ decay. ${ }^{4}$ It needs to be mentioned that these two dimension-6 operators break dark baryon number $B^{\prime}$ and lepton number $L$ respectively. As we will explain later on, they are crucial for $B^{\prime}$ and $L$ asymmetry generation. One of $C P$ phases of parameter $\lambda_{i}$ can be rotated away by field redefinition, and the rest one is the source of $C P$ violation. Coupling parameter $\kappa$ and scale $\Lambda_{1,2}$ can be chosen to be different for different lepton flavor, but here we assume them to be lepton flavor universal for simplicity. For conciseness we do not write out the kinetic terms $\mathcal{L}_{\text {kinetic }}$ in detail.

In the following, we explain how to generate the baryon and dark baryon asymmetries in this model. For convenience, we use " $Y$ " to denote the net number density of a kind of particle relative to entropy density $s$. For selfconjugate particle and nonself-conjugate particle, expressions of $Y$ are slightly different:

$$
\begin{aligned}
Y_{i} & \equiv \frac{n_{i}}{S} \quad(\text { if “ } i \text { " is self-conjugate }) \\
Y_{\Delta i} & \equiv \frac{n_{i}-n_{\bar{i}}}{S} \quad \text { (if " } i \text { " is not self-conjugate) }
\end{aligned}
$$

\footnotetext{
${ }^{3} \mathrm{~A}$ dimension-5 operator $\left(\chi \Phi^{\dagger}\right)(L H)$, which is obtained by integrating out heavy $N_{i}$, can also make $\chi$ decay. But due to the huge hierarchy between $M_{N_{i}}$ and $m_{\chi}$, we assume this operator to be negligible.

${ }^{4}$ By introducing additional new particles and new interactions, we can design a cascade decay chain to make $\chi$ decay without high dimension operator. However, in this work we focus on the study of $\Phi$, and thus we simply use dimension-6 operators to make $\chi$ unstable.
} 


\section{A. Stage I: Out-of-equilibrium and $C P$ violated decay of $N_{1}$}

In this work we consider following hierarchy of particle spectrum:

$$
M_{N_{1}}, \quad M_{N_{2}} \gg m_{\Phi}, \quad m_{\chi} \gg m_{q^{\prime}}, \quad m_{l}
$$

And we further choose $N_{1}$ to be lighter than $N_{2}$, and $\Phi$ to be lighter than $\chi$. For simplicity we assume $N_{1}$ is in the thermal equilibrium when universe temperature $T \gg M_{N_{1}}$. At that temperature, the number density of $N_{1}$ is quantified as:

$$
Y_{N_{1}}=\frac{n_{N_{1}}}{s}=\frac{135 \zeta(3)}{4 \pi^{4} g_{*}} \text {. }
$$

Here $\zeta(3) \simeq 1.2$, and $g_{*}$ is the number of relativistic degree of freedom, which can be approximated to 100 . As the universe temperature decreases, $N_{1}$ starts to decay. If this decay process is out-of-equilibrium and $\lambda_{i}$ have irreducible $C P$ phases, asymmetry between the number density of $\Phi / \chi$ and their antiparticles can be generated $[73,74]$ :

$$
Y_{\Delta \Phi}=-Y_{\Delta \chi}=Y_{N_{1}} \times \epsilon \times \eta
$$

$\epsilon$ in Eq. (5) is the parameter used to describe the $C P$ asymmetry in $N_{1}$ decay:

$$
\epsilon \equiv \frac{\Gamma\left(N_{1} \rightarrow \chi \Phi^{\dagger}\right)-\Gamma\left(N_{1} \rightarrow \bar{\chi} \Phi\right)}{\Gamma\left(N_{1} \rightarrow \chi \Phi^{\dagger}\right)+\Gamma\left(N_{1} \rightarrow \bar{\chi} \Phi\right)}
$$

To obtain a nonzero value of $\epsilon$, the interference between tree level and one-loop level decay amplitudes needs to be calculated. In the case where $M_{N_{2}}$ is much larger than $M_{N_{1}}$, $\epsilon$ can be simply expressed as [75]:

$$
\epsilon \simeq-\frac{3}{16 \pi} \frac{M_{N_{1}}}{M_{N_{2}}} \frac{\operatorname{Im}\left[\left(\lambda_{2}^{*} \lambda_{1}\right)^{2}\right]}{\left|\lambda_{1}\right|^{2}} .
$$

Depending on the sign of $\operatorname{Im}\left[\left(\lambda_{2}^{*} \lambda_{1}\right)^{2}\right], \epsilon$ can be positive or negative.

$\eta$ in Eq. (5) is the efficiency factor that describe the "washout" effects. Asymmetries generated in Eq. (7) can be erased by processes like inverse decay $\left(\chi+\Phi^{\dagger} \rightarrow N_{1}\right)$ or 2to-2 scattering $\left(\bar{q}^{\prime}+l_{R} \rightarrow N_{1}+\bar{\chi}\right)$. If $N_{1}$ is always in the thermal equilibrium, then $\eta$ will be zero. Here we can choose the parameter $\lambda_{1}$ to be small enough, to make the decay width of $N_{1}$ being much smaller than the Hubble expansion rate when temperature is around $M_{N_{1}}$ :

$$
\Gamma_{N_{1}} \ll H\left(T \approx M_{N_{1}}\right)
$$

We can always choose suitable values for $\lambda_{1}$ and $M_{N_{1}}$ that satisfy Eq. (8) and make $N_{1}$ in the thermal equilibrium when $T \gg M_{N_{1}}$. In this case, the value of $\eta$ can be close to 1 .

Before moving to the next stage, here we estimate the scale of $\left|Y_{\Delta \Phi}\right|$ and $\left|Y_{\Delta \chi}\right| . Y_{N_{1}}$ is about $4 \times 10^{-3}$. If we choose $\lambda_{1}$ to be real, $\operatorname{Im}\left(\lambda_{2}^{*}\right)^{2}$ to be $\mathcal{O}(1)$, and $\frac{M_{N_{1}}}{M_{N_{2}}}$ to be $\mathcal{O}(0.01)$, then $\left|Y_{\Delta \Phi}\right|$ and $\left|Y_{\Delta \chi}\right|$ can be as large as $2 \times 10^{-6}$. Smaller $\left|Y_{\Delta \Phi}\right|$ and $\left|Y_{\Delta \chi}\right|$ can be easily obtained by decreasing the value of $\operatorname{Im}\left(\lambda_{2}^{*}\right)^{2}$ or $\frac{M_{N_{1}}}{M_{N_{2}}}$.

\section{B. Stage II: $\Phi$ and $\chi$ decay to leptons and dark quarks}

In the Lagrangian we introduce two operators of $\chi$ to break $B^{\prime}$ and $L$ conservation. $\left(\bar{q}^{\prime C} \chi\right)\left(\bar{q}_{L}^{\prime C} l_{R}\right)$ breaks $B^{\prime}$ number by 1 , and $\left(\bar{\chi} \gamma^{\mu} q^{\prime}\right)\left(\bar{d}_{R} \gamma_{\mu} u_{R}\right)$ breaks $L$ number by 1. Both operators are necessary for the generation of baryon and dark baryon asymmetries. This is because $\Phi$ and $\chi$ carry the same particle number, but after $N_{1}$ decay we obtain $Y_{\Delta \Phi}=-Y_{\Delta \chi}$. So the net particle number density of $q^{\prime}$ and $l$ can not be generated without $B^{\prime}$ and $L$ violated processes. We denote the branching ratios of decay channels $\chi \rightarrow \bar{q}^{\prime} q^{\prime} \bar{l}_{R}$ and $\chi \rightarrow q^{\prime} \bar{d}_{R} u_{R}$, by $B r_{\chi}\left(B^{\prime}\right)$ and $B r_{\chi}(\not L)$ respectively. Because $\chi$ only has two decay channels, so we should have $B r_{\chi}\left(\boldsymbol{B}^{\prime}\right)+B r_{\chi}(\not{L})=1$.

We need the sphaleron process in the SM to transfer lepton number to baryon number. Lattice simulation shows that the SM sphaleron transition rate will be smaller than the Hubble expansion rate when the universe temperature is $T_{*}=(131.7 \pm 2.3) \mathrm{GeV}[76]$. So $\Phi$ and $\chi$ need to decay before $T_{*}$. This requirement can be satisfied by choosing $m_{\Phi}$ and $m_{\chi}$ to be higher than $T_{*}$, and make their lifetime shorter than $1 / H\left(T_{*}\right)$. If we assume that the net number densities of $\Phi$ and $\chi$ have been almost entirely transferred to the number densities of $l_{R}$ and $q^{\prime}$ before $T_{*}$. Then there will be $\mathrm{b}^{5}$

$$
\begin{aligned}
Y_{\Delta l_{R}} \simeq-Y_{\Delta \Phi}-B r_{\chi}\left(\boldsymbol{B}^{\prime}\right) \times Y_{\Delta \chi}=-B r_{\chi}(\not \mathcal{L}) \times Y_{\Delta \Phi} \\
Y_{\Delta q^{\prime}} \simeq Y_{\Delta \Phi}+\left(B r_{\chi}(\not \boldsymbol{Z})-2 B r_{\chi}\left(\boldsymbol{B}^{\prime}\right)\right) \times Y_{\Delta \chi} \\
=3 B r_{\chi}\left(\boldsymbol{B}^{\prime}\right) \times Y_{\Delta \Phi}
\end{aligned}
$$

\section{Stage III: Generate baryon and dark baryon asymmetries}

Each dark quark $q^{\prime}$ carries $1 / 3$ dark baryon number, thus after $\Phi$ and $\chi$ decay we obtain:

\footnotetext{
${ }^{5}$ Here we also require decay processes $\chi \rightarrow \bar{q}^{\prime} q^{\prime C} \bar{l}_{R}$ and $\chi \rightarrow$ $q^{\prime} \bar{d}_{R} u_{R}$ to be out-of-equilibrium. Otherwise the decay products of these two processes will transfer to each other, through $\bar{q}^{\prime} q^{\prime} \bar{l}_{R} \leftrightarrow \chi \leftrightarrow q^{\prime} \bar{d}_{R} u_{R}$. And thus the estimation of particle number density will be more complicated.
} 


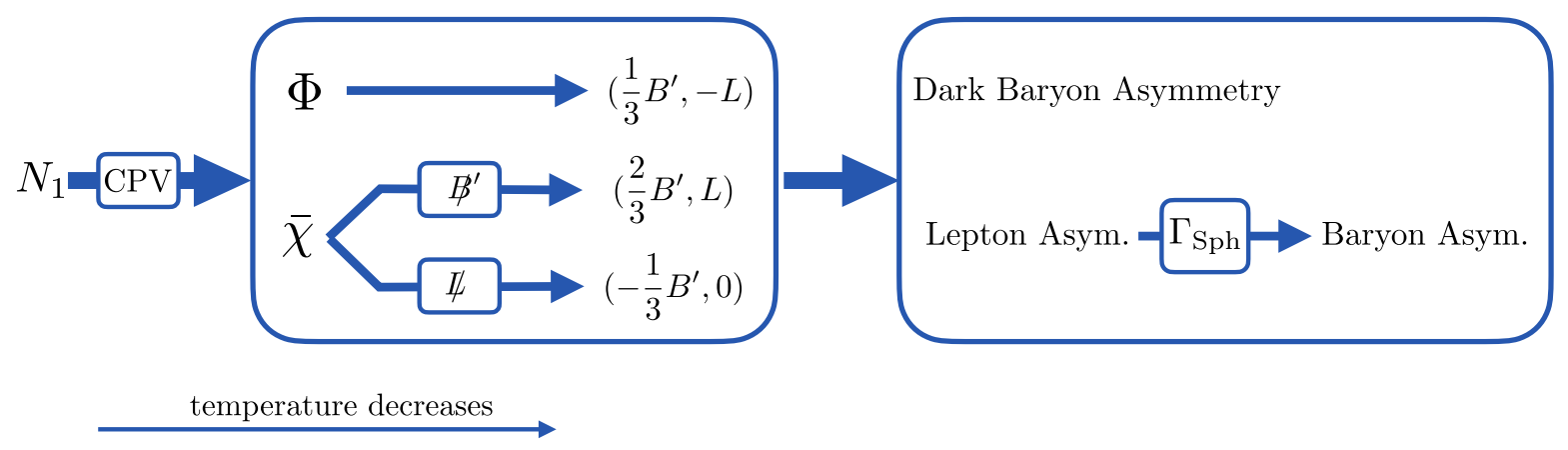

FIG. 1. A schematic diagram for the DM asymmetry and baryon asymmetry generation process. Firstly, the out-of-equilibrium and $C P$ violated decay of $N_{1}$ generates the asymmetries of $\Phi$ and $\chi$. Then the decay of $\Phi$ and $\chi$ generate the asymmetries of dark baryon and lepton. Finally, the lepton asymmetry is transferred to baryon asymmetry via sphaleron process.

$$
Y_{\Delta B^{\prime}}=\frac{1}{3} Y_{\Delta q^{\prime}} \simeq B r_{\chi}\left(\boldsymbol{B}^{\prime}\right) \times Y_{\Delta \Phi}
$$

On the other hand, gauge interaction, Yukawa interaction, and sphaleron transition in the SM are rapid when the universe temperature is higher than $T_{*}$. A combination of all these processes finally transfer the lepton number, which are carried by $l_{R}$, to baryon number [77]:

$Y_{\Delta B}=\frac{28}{79} Y_{\Delta B-L}=-\frac{28}{79} Y_{\Delta l_{R}} \simeq \frac{28}{79} B r_{\chi}(\not L) \times Y_{\Delta \Phi}$

As explained in stage I, $\left|Y_{\Delta \Phi}\right|$ can be as large as $2 \times 10^{-6}$, and thus it is easy to explain the observed BAU $Y_{\Delta B} \approx 8 \times 10^{-11}$, provided $B r_{\chi}(\not L)$ is not too small.

The mass of dark baryon can be fixed by the ratio between $Y_{\Delta B^{\prime}}$ and $Y_{\Delta B}$ :

$$
\frac{m_{B^{\prime}} Y_{\Delta B^{\prime}}}{m_{B} Y_{\Delta B}}=\frac{\Omega_{\mathrm{DM}}}{\Omega_{B}} \Rightarrow m_{B^{\prime}} \simeq \frac{B r_{\chi}(\not \not)}{B r_{\chi}\left(\not^{\prime}\right)} \times 1.67 \mathrm{GeV}
$$

So in this model, dark baryon mass relies on the branching ratios of $\chi$, and can vary in a wide range. In Fig. 1 we present a schematic diagram to summarizes above asymmetry generation process.

\section{Stability of dark baryon $\boldsymbol{B}^{\prime}$ and dark pion $\boldsymbol{\pi}^{\prime}$}

$\chi$ in Lagrangian (1) couples to a $B^{\prime}$ violated operator and a $L$ violated operator, and thus a dark baryon (composed by $\left.q^{\prime} q^{\prime} q^{\prime}\right)$ can decay to $l^{+} \pi^{-}$via $\chi$. On the other hand, dark pion (composed by $\bar{q}^{\prime} q^{\prime}$ ) can decay to $l^{+} l^{-}$via a t-channel $\Phi$. In Fig. 2 we present these two decay processes for illustration.

By integrating out $\chi$ and $\Phi$ in the Lagrangian, we obtain a dimension-9 operator and a dimension- 6 operator that induce $B^{\prime}$ and $\pi^{\prime}$ decay respectively:

$$
\begin{aligned}
\mathcal{L} \supset & \frac{\kappa^{2}}{m_{\Phi}^{2}}\left(\bar{q}_{L}^{\prime} l_{R}\right)\left(\bar{l}_{R} q_{L}^{\prime}\right) \\
& +\left[\frac{1}{m_{\chi} \Lambda_{1}^{2} \Lambda_{2}^{2}}\left(\bar{q}_{L}^{\prime C} l_{R}\right)\left(\bar{q}^{\prime C} \gamma^{\mu} q^{\prime}\right)\left(\bar{d}_{R} \gamma_{\mu} u_{R}\right)+\text { H.c. }\right]
\end{aligned}
$$

Thus the decay width of $B^{\prime}$ is suppressed by the factor $m_{\chi}^{2} \Lambda_{1}^{4} \Lambda_{2}^{4}$. Current bound on dark matter lifetime from weak lensing and cluster counts is $\tau_{B^{\prime}} \gtrsim 175 \mathrm{Gyr}$ [78]. This bound can be rewritten as $\Gamma\left(B^{\prime}\right) \lesssim 1.2 \times 10^{-43} \mathrm{GeV}$. Dimensional analysis says $\Gamma\left(B^{\prime}\right) \simeq \frac{1}{8 \pi} \frac{m_{B^{\prime}}^{11}}{m_{\chi}^{2} \Lambda_{1}^{4} \Lambda_{2}^{4}}$. Thus we can simply put $m_{\chi}$ and $\Lambda_{1,2}$ to a scale higher than $20 \mathrm{TeV}$, which is unreachable for current particle physics experiments, to satisfy the lifetime bound. In the following sections we will not discuss the detection of $\chi$.

In our ADM scenario, anti-DM $\bar{B}^{\prime}$ in the early universe will be almost completely wiped out via the annihilation with $\mathrm{DM} B^{\prime}$. And thus the annihilation process $B^{\prime} \bar{B}^{\prime} \rightarrow \pi^{\prime} \pi^{\prime}$ produce plenty of dark meson $\pi^{\prime}$ in the early universe. If those $\pi^{\prime}$ are long-lived like $B^{\prime}$, they will overclose the
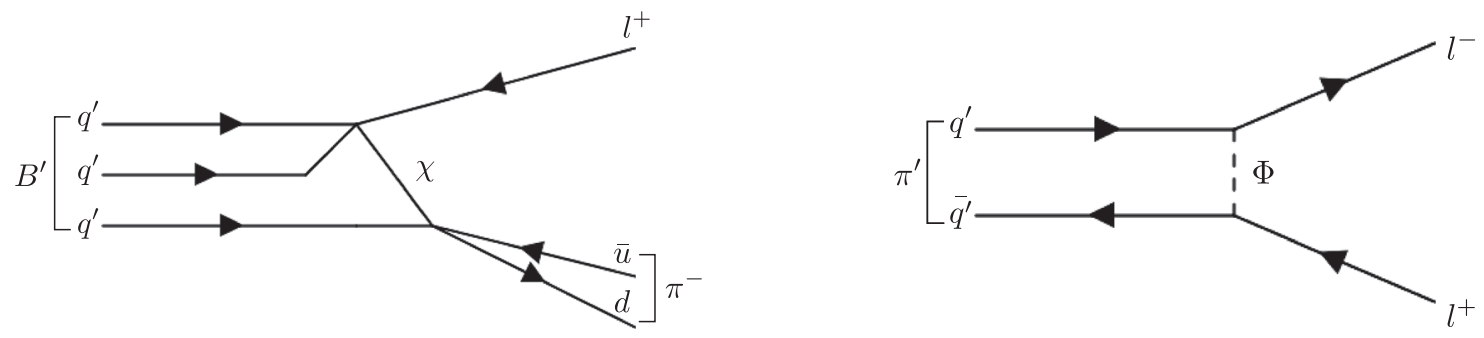

FIG. 2. Left: illustration for dark baryon $B^{\prime}$ decaying to $l^{+} \pi^{-}$via $\chi$. Right: illustration for dark pion $\pi^{\prime}$ decaying to $l^{+} l^{-}$via $\Phi$. 
Universe (if $\pi^{\prime}$ is heavy) or affect the effective number of neutrino degrees of freedom $N_{\text {eff }}$ (if $\pi^{\prime}$ is light) [44]. So $\pi^{\prime}$ should decay before BBN. Through the dimension-6 operator in Lagrangian (14) we obtain the coupling between $\pi^{\prime}$ and lepton pair, $\frac{i \kappa^{2} f_{\pi^{\prime}} m_{l}}{m_{\Phi}^{2}} \pi^{\prime} \bar{l}_{R} \gamma_{5} l_{R}$ [45]. Thus the decay width of $\pi^{\prime} \rightarrow \bar{l} l$ is [58]:

$\Gamma\left(\pi^{\prime} \rightarrow \bar{l} l\right)=\frac{\kappa^{4}}{32 \pi m_{\Phi}^{4}} f_{\pi^{\prime}}^{2} m_{l}^{2} m_{\pi^{\prime}}, \quad$ with $\quad l=e, \mu, \tau$

Here $f_{\pi^{\prime}}$ is the decay constant of dark pion $\pi^{\prime}$. Due to the hierarchy between $m_{e}, m_{\mu}$, and $m_{\tau}$, the lifetime of $\pi^{\prime}$ is determined by the decay width to the heaviest lepton pair allowed by kinematics. The constraint from BBN give us a bound on $\pi^{\prime}$ lifetime:

$$
\begin{aligned}
\frac{1}{\Gamma\left(\pi^{\prime} \rightarrow \bar{l} l\right)} & \lesssim 1.52 \times 10^{24} \mathrm{GeV}^{-1} \\
& \Rightarrow m_{\Phi} \lesssim 0.35 \times 10^{6} \times \kappa \sqrt{f_{\pi^{\prime}} m_{l}} \times\left(\frac{m_{\pi^{\prime}}}{1 \mathrm{GeV}}\right)^{\frac{1}{4}}
\end{aligned}
$$

If we choose a benchmark setting like: $\kappa=1, f_{\pi^{\prime}}=$ $m_{\pi^{\prime}}=0.3 \mathrm{GeV}$. Then $\pi^{\prime}$ can only decay to $\bar{\mu} \mu$ and the up-limit of $m_{\Phi}$ from BBN is $2.6 \times 10^{5} \mathrm{GeV}$. If the dark confinement scale $\Lambda^{\prime}$ become higher and $m_{\pi^{\prime}}$ can decay to $\bar{\tau} \tau$, then the upper limit of $m_{\Phi}$ can be increased by more than an order of magnitude.

On the other hand, $\Phi$ can not be lighter than the sphaleron freeze-out temperature $T_{*} \approx 131.7 \mathrm{GeV}$, because we need to generate enough antileptons via $\Phi$ decay, before the sphaleron process stops. We assume the coupling $\kappa$ for interaction $\Phi \bar{q}_{L}^{\prime} l_{R}$ is not too small, and thus $\Phi$ is always in the thermal bath during its decay. Then the proportion of $\Phi$ particles that have decayed before $T_{*}$ can be estimated by $1-e^{-m_{\Phi} / T_{*}}$. So even $\Phi$ is as light as $300 \mathrm{GeV}, 90 \%$ of $\Phi$ would have decayed before Sphaleron process stop. This is enough to explain BAU in a large parameter space of this model.

Such a small mass lower-limit makes scalar mediator $\Phi$ a promising target for current and coming particle physics experiments. In the following sections, we will discuss direct detection and collider detection of this model, through the portal provided by scalar mediator $\Phi$. To simplify our analysis, in this work we will assume that $B r_{\chi}(\not Z)$ and $B r_{\chi}\left(\boldsymbol{B}^{\prime}\right)$ are in the same order of magnitude, and fix $m_{B^{\prime}}$ to $2 \mathrm{GeV}$. This choice also fix the value of dark confinement scale: $\Lambda_{\mathrm{QCD}}^{\prime} \simeq \frac{m_{B^{\prime}}}{m_{B}} \Lambda_{\mathrm{QCD}} \simeq 0.64 \mathrm{GeV}$.

\section{DIRECT DETECTION}

In this section we discuss the direct detection of this model. Dark baryon $B^{\prime}$, which is composed by 3 dark quarks, can scatter with electrons in atoms through the dimension-6 operator:

$$
\frac{\kappa^{2}}{m_{\Phi}^{2}}\left(\bar{q}_{L}^{\prime} e_{R}\right)\left(\bar{e}_{R} q_{L}^{\prime}\right)=\frac{\kappa^{2}}{2 m_{\Phi}^{2}}\left(\bar{q}_{L}^{\prime} \gamma^{\mu} q_{L}^{\prime}\right)\left(\bar{e}_{R} \gamma_{\mu} e_{R}\right)
$$

Here we use Fierz identity to rewrite the operator. So it is possible to detect $B^{\prime}$ by direct detection experiments via electron ionization.

The matrix element for the dominant spin-independent scattering is [79]:

$$
\mathcal{M}=\frac{\kappa^{2}}{8 m_{\Phi}^{2}} g_{\mu \nu} J_{B^{\prime}}^{\mu} J_{e}^{\nu}
$$

where $J_{e}^{\nu}=\bar{u}\left(p^{\prime}\right) \gamma^{\nu} u(p)$, and $J_{B^{\prime}}^{\mu}=\left\langle B^{\prime}\left(k^{\prime}\right)\left|\overline{q^{\prime}} \gamma^{\mu} q^{\prime}\right| B^{\prime}(k)\right\rangle \approx$ $3 \bar{u}\left(k^{\prime}\right) \gamma^{\mu} u(k)$, in the nonrelativistic limit. Here $k(p)$ and $k^{\prime}$ $\left(p^{\prime}\right)$ are initial and final state momentum of $B^{\prime}$ (electron) respectively. So the spin-independent cross section of $B^{\prime}$-electron scattering is

$$
\bar{\sigma}_{e B^{\prime}} \approx \frac{9 \kappa^{4} \mu_{e B^{\prime}}^{2}}{64 \pi m_{\Phi}^{4}}
$$

Here $\mu_{e B^{\prime}}=\left(m_{e} m_{B^{\prime}}\right) /\left(m_{e}+m_{B^{\prime}}\right)$ is the reduced mass. If we choose $\kappa=1$ and $m_{\Phi}=300 \mathrm{GeV}$, then $\bar{\sigma}_{e B^{\prime}} \approx$ $5.6 \times 10^{-46} \mathrm{~cm}^{2}$. This is much smaller than current direct detection limits from XENON100 [80,81] or DarkSide-50 [82]. On the other hand, dark baryon $B^{\prime}$ can also scatter with nucleons via loop induced process. But the corresponding DM-nucleon scattering cross section will be suppressed by loop factor. Furthermore, direct detection bounds on DM-nucleon scattering sharply become loose when DM mass is lighter than $10 \mathrm{GeV}$. So here we conclude that current direct detection experiments can hardly constrain our model, provided $m_{B^{\prime}}$ is around $\mathrm{GeV}$ scale.

\section{COLLIDER SEARCH}

In this section we study the detection of this model by collider experiments, including both hadron collider (e.g., LHC) and lepton collider (e.g., CEPC). The Lagrangian which is related to collider search can be expressed as [58]:

$$
\begin{aligned}
\mathcal{L} \supset \bar{q}^{\prime}\left(\not D-m_{q^{\prime}}\right) q^{\prime}+\left(D_{\mu} \Phi\right)^{\dagger}\left(D^{\mu} \Phi\right)-m_{\Phi}^{2} \Phi^{\dagger} \Phi \\
-\frac{1}{4} G^{\prime \mu \nu} G_{\mu \nu}^{\prime}-\left(\kappa \Phi \bar{q}_{L}^{\prime} l_{R}+\text { H.c. }\right)
\end{aligned}
$$

Here $G^{\prime \mu \nu}$ is the field strength of dark gluon. If dark quark $q^{\prime}$ can be produced at colliders via dimension- 6 operator or $\Phi$ decay, then generally it will carry an energy which is much larger than the dark confinement scale $\Lambda_{\mathrm{QCD}}^{\prime}$ (in Sec. II D we have fixed $\Lambda_{\mathrm{QCD}}^{\prime}$ to $0.64 \mathrm{GeV}$ ). Thus the energetic $q^{\prime}$ will shower then evolve to a bunch of collinear dark mesons after hadronization. Such an object is often 
call "dark jet." Dark jet is not totally invisible at collider since dark meson can decay back to SM particles through some portal. As we have explained in Sec. II D, dark meson $\pi^{\prime}$ can decay to lepton pair via the dimension-6 operator provided by $\Phi$. Here we estimate how long it can propagate before it decay. Proper lifetime of dark meson $\pi^{\prime}$ is [58]:

$$
\begin{aligned}
c \tau_{0}= & \frac{c \hbar}{\Gamma_{\pi^{\prime}}} \approx 120 \mathrm{~mm} \times \frac{1}{\kappa^{4}}\left(\frac{1 \mathrm{GeV}}{f_{\pi^{\prime}}}\right)^{2}\left(\frac{0.1 \mathrm{GeV}}{m_{l}}\right)^{2} \\
& \times\left(\frac{1 \mathrm{GeV}}{m_{\pi^{\prime}}}\right)\left(\frac{m_{\Phi}}{500 \mathrm{GeV}}\right)^{4} .
\end{aligned}
$$

So we can expect that, in a large parameter space of this model, dark mesons inside dark jet can propagate for a moment, and then decay to lepton pair at a place away from the primary vertex. Such a novel "displaced lepton jet" is the main signal for the collider searches of this model.

To simplify our collider analysis, we fix the mass of $\pi^{\prime}$ to $0.3 \mathrm{GeV}$, and thus the dominant decay channel of $\pi^{\prime}$ is $\pi^{\prime} \rightarrow \bar{\mu} \mu$. Furthermore, we choose the proper lifetime of $\pi^{\prime}$, $c \tau_{0}$, to be input parameter instead of $f_{\pi^{\prime}}$. Because $c \tau_{0}$ is directly related to collider phenomenology. $\kappa$ and $m_{\Phi}$ are other two input parameters for collider search.

For Monte Carlo simulation, we use FeynRules [83] to write Lagrangian (20) into an UFO model file [84]. Parton level events are generated by MadGraph5 [85], then showered and hadronized by PYTHIA8 [86]. The HIDDENVALLEY [50] module implemented in PYTHIA8 can be used to simulate dark shower and dark hadronization process. Detector simulation is performed by DELPHES3 [87]. Finally, we use the anti-kt algorithm [88] implemented in FASTJET [89] to do jet clustering if needed.

\section{A. Detection at LHC}

ATLAS group already performed the displaced lepton jets signal search at $13 \mathrm{TeV}$ LHC by using an event sample of integrated luminosity $3.4 \mathrm{fb}^{-1}$ [90]. Deviations from the SM expectations are not observed. In this subsection we will use the results presented in [90] to constrain our model.

Scalar mediator $\Phi$ carries Hyper charge " +1 " and dark $S U(3)^{\prime}$ charge. Thus the cross section of $\Phi$ pair production is just three times that of right-handed slepton pair production, when their masses are the same. NLO+ NLL cross section of slepton pair production at $13 \mathrm{TeV}$ LHC have been given in [91]. Based on their results, in Fig. 3 we present the cross section of $\Phi$ pair production at $13 \mathrm{TeV}$ LHC. It can be seen that this cross section is quite small. For example, if $m_{\Phi}$ is $300 \mathrm{GeV}$, then integrated luminosity $3.4 \mathrm{fb}^{-1}$ can only generate 17.6 signal events. Event preselection in [90] will eliminate more than half of the signal events. After that, depending on the position where long-lived particle decay, the tagging efficiency of displaced lepton jets varies from 50\% to $10 \%$. And the signal region require two displaced lepton jets. Thus the

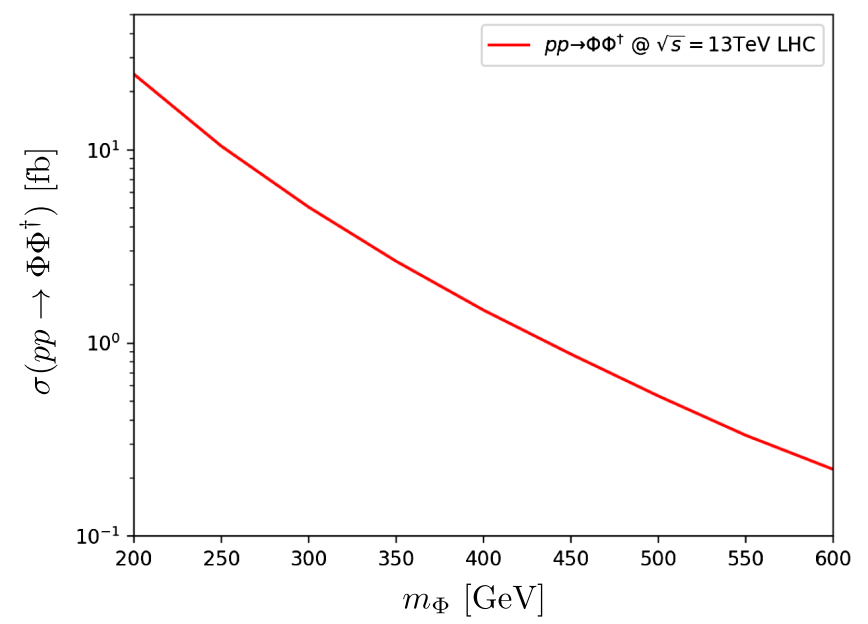

FIG. 3. Cross section of $\Phi$ pair production process at $13 \mathrm{TeV}$ LHC as a function of $m_{\Phi}$.

original number of signal events will be suppressed by about an order, after performing the full cut flow in [90]. On the other hand, the irreducible background for displaced muon jets, which mainly comes from cosmic-rays, is expected to be $31.8 \pm 3.8$ (stat) \pm 8.6 (syst). And experiment [90] finally record 46 events. So we can conclude that our model is very difficult to be detected or excluded by current LHC data.

\section{B. Detection at lepton collider}

Different with hadron collider, projected lepton collides, including ILC [92], CLIC [93], FCC-ee [94], and CEPC [72], use electron and positron as injecting beam. Thus it is possible to directly generate dark quark pair through a t-channel process, see Fig. 4 (left) for illustration. When the mass of mediator $\Phi$ is large enough, we can integrate $\Phi$ and obtain an dimension- 6 operator:

$$
\frac{\kappa^{2}}{m_{\Phi}^{2}}\left(\bar{q}_{L}^{\prime} e_{R}\right)\left(\bar{e}_{R} q_{L}^{\prime}\right)
$$

Thus the cross section of $e^{+} e^{-} \rightarrow \bar{q}^{\prime} q^{\prime}$ can be approximated as:

$$
\sigma\left(e^{+} e^{-} \rightarrow \bar{q}^{\prime} q^{\prime}\right) \approx \frac{\kappa^{4}}{256 \pi} \frac{s}{m_{\Phi}^{4}}
$$

where electron and $q^{\prime}$ are treated as massless. In the rest part of this subsection, we will choose CEPC, with central energy $\sqrt{s}=240 \mathrm{GeV}$ and integrated luminosity $5.6 \mathrm{ab}^{-1}$, as a benchmark setting for lepton collider detection.

In Fig. 4 (right) we present $\sigma\left(e^{+} e^{-} \rightarrow \bar{q}^{\prime} q^{\prime}\right)$ as functions of $m_{\Phi}$ with $\kappa$ fixed to 0.5 and 1.0. Thanks to the expected high luminosity, CEPC can produce plenty of $q^{\prime}$ pair even with $\mathrm{TeV}$ scale mediator. Dark quark $q^{\prime}$ finally evolve to a jet-like object, which is composed by lots of displaced 

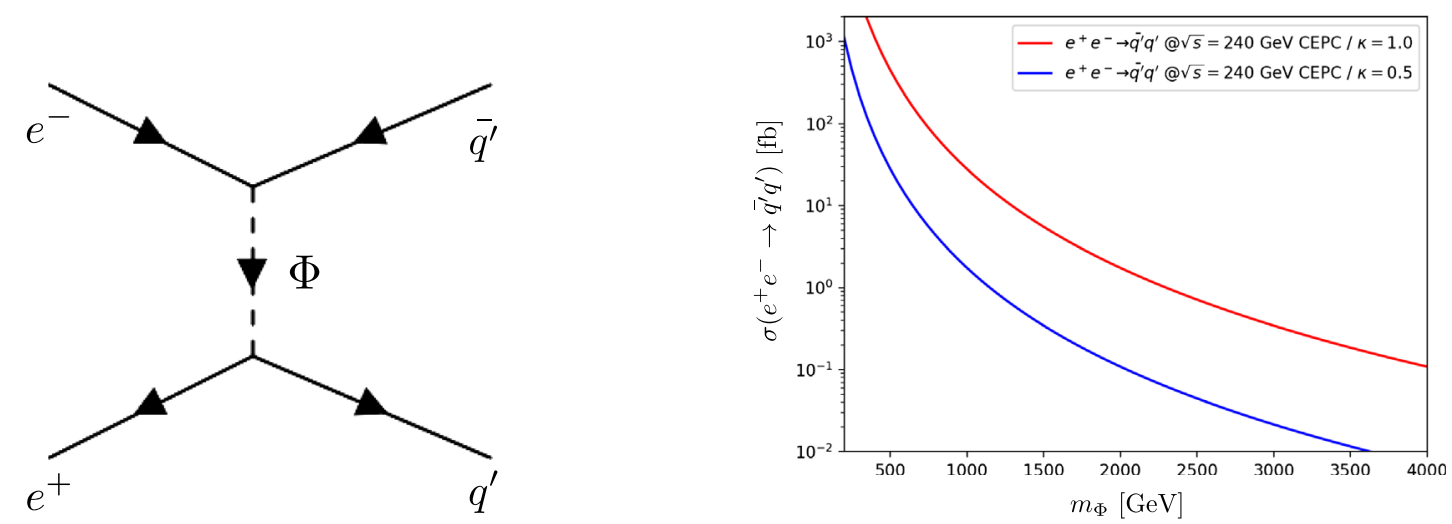

FIG. 4. Left: Feynman diagram of the signal process at CEPC. Right: Cross section of $q^{\prime}$ pair production process at CEPC as functions of $m_{\Phi}$, with coupling $\kappa$ fixed to 0.5 and 1.0 respectively.
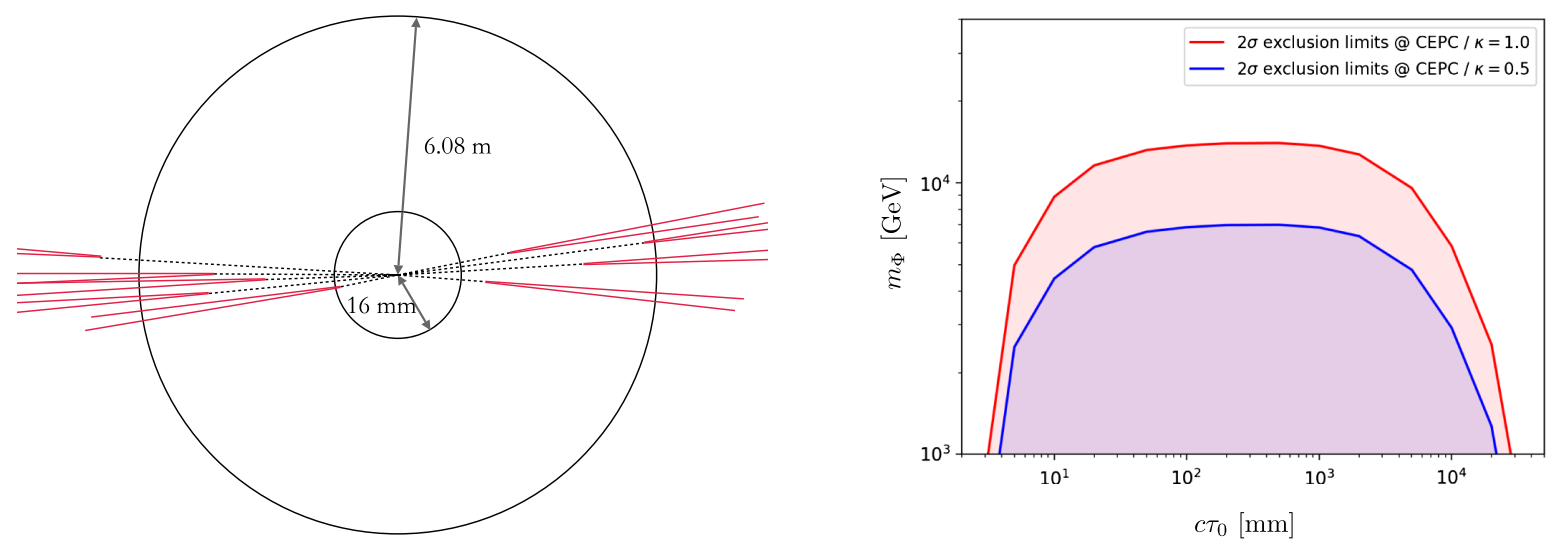

FIG. 5. Left: Illustration plot of the signal process at CEPC. Detector size is denoted by two circles. Black dotted lines and red solid lines are dark pions and muons, respectively. Right: $2 \sigma$ exclusion limits on mediator mass $m_{\Phi}$ as functions of the dark pion proper decay length, with coupling $\kappa$ fixed to 0.5 and 1.0 respectively.

muons, see Fig. 5 (left) for illustration. Detection of displaced signals is closely related to the physical size of detector. For CEPC, the inner radius of inner tracker (IT) is $16 \mathrm{~mm}$, and the outer radius of muon system (MS) is $6.08 \mathrm{~m}$. Based on these design information, here we propose a cut-flow to search for displaced lepton jets:

(i) Dark pion $\pi^{\prime}$ decay at a place away from primary vertex, and then two daughter muons come out and leave tracks in detectors. Thus it is possible to reconstruct the displaced vertex (DV) from $\pi^{\prime}$ decay via daughter muons' tracks. Detailed discussion on DV tagging is given in the Appendix. All the muons that can be traced back to a DV will be labelled as displaced muon. ${ }^{6}$

\footnotetext{
${ }^{6}$ Our definition of displaced muon is slightly different with the conventional definition of displaced lepton which is based on impact parameter. This definition helps to reduce the contamination from cosmic ray. See the discussion in the following paragraph.
}

(ii) We use all the displaced muons, with $p_{\mathrm{T}}>1 \mathrm{GeV}$ and $|\eta|<3.0$, as input of jet clustering. We use antikt algorithm with jet radius $R=0.4$ to do jet clustering. If there are 6 , or more than 6 , displaced muons inside a jet, then this jet will be tagged as a displaced muon jet (DMJ).

(iii) For a signal event, we require the number of DMJs to be greater than 2 .

Unlike the ATLAS DMJ search which is designed for Falkowsky-Ruderman-Volansky-Zupan (FRVZ) model $[95,96]$ and only require at least two muons inside a DMJ, our CEPC DMJ criteria require much more displaced muons inside it. Thus our DMJ can not be faked by SM background processes. Furthermore, all the displaced muons can be paired by the DV which they belong to, and the reconstructed momentum of each displaced muon pair should point to the primary vertex. There are at least six displaced muon pair in our signal event. These amount of muon pairs, with their momentums point to the primary vertex, can hardly be faked by cosmic rays. So the search of our DMJ signal at CEPC can be treated as background free $[97,98]$. 
If there is no background events, then the $2 \sigma$ exclusion limit correspond to 3 expected signal events. This is called "rule of three" is statistics. In Fig. 5 (right) we present the $2 \sigma$ exclusion limits on $c \tau_{0}-m_{\Phi}$ plane with $\kappa$ fixed to 0.5 and 1.0. In this exclusion plot we choose central energy $\sqrt{s}=240 \mathrm{GeV}$ and integrated luminosity $5.6 \mathrm{ab}^{-1}$ for CEPC setting. It can be seen that $m_{\Phi}$ can be excluded up to about $10 \mathrm{TeV}$ scale at future CEPC, provided the proper lifetime of dark pion is in the range of $10 \mathrm{~mm}$ to $10 \mathrm{~m}$, which just correspond to the physical size of CEPC detector.

Before we finish this section, we give a brief discussion on displaced electron jet signal. If we reduce the mass of $\pi^{\prime}$ to less than $200 \mathrm{MeV}$, then the main decay channel of $\pi^{\prime}$ will be $\pi^{\prime} \rightarrow \bar{e} e$, and thus the collider signature for our model changes to displaced electron jets. At LHC, the displaced electron jets signal suffer from multijets contamination, and ATLAS observed 239 displaced electron jet pair events that mainly comes from multijets process [90]. So the detection of our model at LHC will become more difficult if the signal is displaced electron jets. CEPC is much more cleaner than LHC, and we can expect that the contamination from multijets process at CEPC is less serious than those at LHC. Detailed detector level analysis is required to make it clear. We leave it for a future study.

\section{CONCLUSION}

In this work we propose a composite asymmetric dark matter model. The dark sector in this model talks to the SM sector through a scalar mediator, which couples to a SM lepton and a dark quark. This model can successfully explain the observed baryon asymmetry of the universe and dark matter relic density. Detection of this model is briefly discussed. We find that current dark matter direct detection, via DM-electron scattering or DM-nucleon scattering, can hardly constrain this model. Furthermore, due to the small production cross section and irreducible background, current LHC data is also incapable to detect this model. Finally, we find it is promising to detect this model by "displaced lepton jets" signal at future lepton colliders. Using CEPC as a representative lepton collider, we find that CEPC could exclude the mass of mediator up to $10 \mathrm{TeV}$ scale, provided the proper lifetime of dark pion varies from $10 \mathrm{~mm}$ to $10 \mathrm{~m}$.

\section{ACKNOWLEDGMENTS}

M.Z. thanks Junmou Chen, Zhen Liu, Michael J. Ramsey-Musolf, Manqi Ruan, and Fanrong Xu for useful discussions. M.Z. appreciates Fa Peng Huang for careful reading of this manuscript. This work was supported by the National Natural Science Foundation of China (NNSFC) under Grant No. 11947118.
TABLE II. Physical size and spatial resolution of different detectors on CEPC. Here $R_{\text {in }}, R_{\text {out }}, \sigma_{x y}$, and $\sigma_{z}$ are inner radius, outer radius, transverse spatial resolution, and longitudinal spatial resolution of different detectors respectively.

\begin{tabular}{lcccc}
\hline \hline Detector & $R_{\text {in }}$ & $R_{\text {out }}$ & $\sigma_{x y}$ & $\sigma_{z}$ \\
\hline Vertex detector & $16 \mathrm{~mm}$ & $60 \mathrm{~mm}$ & $(2.8 \sim 6) \mu \mathrm{m}$ & $(2.8 \sim 6) \mu \mathrm{m}$ \\
Silicon tracker & $0.15 \mathrm{~m}$ & $1.81 \mathrm{~m}$ & $7.2 \mu \mathrm{m}$ & $86.6 \mu \mathrm{m}$ \\
Hadron calorimeter & $2.30 \mathrm{~m}$ & $3.34 \mathrm{~m}$ & $30 \mathrm{~mm}$ & $30 \mathrm{~mm}$ \\
Muon system & $4.40 \mathrm{~m}$ & $6.08 \mathrm{~m}$ & $2.0 \mathrm{~cm}$ & $1.5 \mathrm{~cm}$ \\
\hline \hline
\end{tabular}

\section{APPENDIX: DISPLACED VERTEX TAGGING AT CEPC}

As we explained in the main text, dark pion $\pi^{\prime}$ is long-lived and can probably leave displaced vertex (DV) inside the detector of CEPC. In real experiment, the reconstruction of DV closely depend on the physical size and spatial resolution of the detector. We briefly present those information of detectors on CEPC in Table II [72].

By using all the information in the tracking system, CEPC angular resolution for tracks can be better than $10^{-3} \mathrm{rad}$, provided the $p_{\mathrm{T}}$ of tracks are larger than $1 \mathrm{GeV}$ [72]. However, this angular resolution is only valid for those tracks coming from the primary vertex. Here we make an estimation for the angular resolution of tracks (left by two daughter muons) coming from DV.

We denote the transverse distance from a DV to the primary vertex as $L_{x y}$. We only consider those DV within the range $16 \mathrm{~mm}<L_{x y}<5.0 \mathrm{~m}$. Now we consider the DV with $L_{x y}=5.0 \mathrm{~m}$. Based on the design information present in Table II, we know that there will be two daughter muons

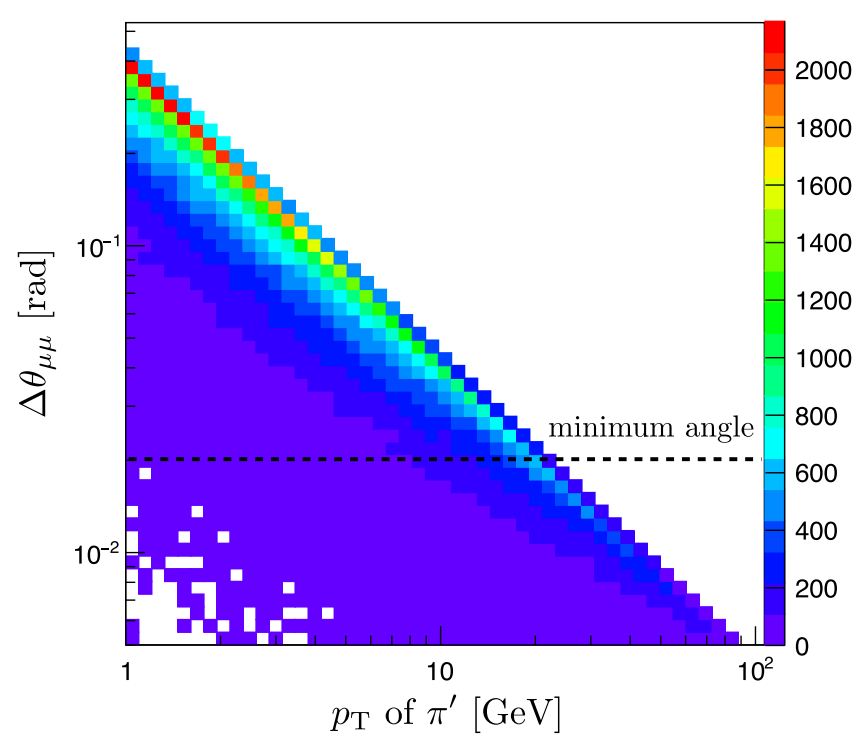

FIG. 6. Distribution of $\Delta \theta_{\mu \mu}$ and $p_{\mathrm{T}}$ of $300,000 \pi^{\prime}$ produced by dark hadronization process at CEPC. 
propagate in the Muon System and leave tracks longer than $1 \mathrm{~m}$. On the other hand, the spatial resolution of Muon System is $2.0 \mathrm{~cm}$. Here we denote the angle between two daughter muons as $\Delta \theta_{\mu \mu}$. We can expect that the mother DV can be reconstructed if $\Delta \theta_{\mu \mu}>0.02 \mathrm{rad}$ (which is just $\left.\frac{2 \mathrm{~cm}}{1 \mathrm{~m}}=\frac{\text { spatial resolution }}{\text { track length }}\right)$. For the DV with $L_{x y}<5.0 \mathrm{~m}$, we can utilize longer track and smaller spatial resolution, and thus the minimum value of $\Delta \theta_{\mu \mu}$ can be even smaller. For simplicity, in this work we only require $\Delta \theta_{\mu \mu}>0.02 \mathrm{rad}$ for DV reconstruction.
If the sub-GeV dark pion $\pi^{\prime}$ produced at CEPC is highly boosted, then the corresponding $\Delta \theta_{\mu \mu}$ will be too small for DV reconstruction. But thanks to the dark hadronization, the energy of the original dark quark is shared by multiple dark pion $\pi^{\prime}$. In Fig. 6 we show the distribution of $\Delta \theta_{\mu \mu}$ and $p_{\mathrm{T}}$ of 300,000 generated $\pi^{\prime}$. It can be seen that the $p_{\mathrm{T}}$ of most $\pi^{\prime}$ are smaller than $10 \mathrm{GeV}$, and the corresponding $\Delta \theta_{\mu \mu}$ are generally larger than $0.02 \mathrm{rad}$. Thus most of the decayed $\pi^{\prime}$ can be tagged as DV, provided the proper lifetime of $\pi^{\prime}$ is within the size of CEPC detector.
[1] N. Aghanim et al. (Planck Collaboration), Planck 2018 results. I. Overview and the cosmological legacy of Planck, Astron. Astrophys. 641, A1 (2020).

[2] B. D. Fields, K. A. Olive, T. H. Yeh, and C. Young, Bigbang nucleosynthesis after Planck, J. Cosmol. Astropart. Phys. 03 (2020) 010; Erratum, J. Cosmol. Astropart. Phys. 11 (2020) E02.

[3] A. D. Sakharov, Violation of $C P$ invariance, C asymmetry, and baryon asymmetry of the universe, Sov. Phys. Usp. 34, 392 (1991).

[4] V. A. Kuzmin, V. A. Rubakov, and M. E. Shaposhnikov, On the anomalous electroweak baryon number nonconservation in the early universe, Phys. Lett. 155B, 36 (1985).

[5] M.E. Shaposhnikov, Possible appearance of the baryon asymmetry of the universe in an electroweak theory, JETP Lett. 44, 465 (1986).

[6] M. E. Shaposhnikov, Baryon Asymmetry of the universe in standard electroweak theory, Nucl. Phys. B287, 757 (1987).

[7] M. Fukugita and T. Yanagida, Baryogenesis without grand unification, Phys. Lett. B 174, 45 (1986).

[8] I. Affleck and M. Dine, A new mechanism for baryogenesis, Nucl. Phys. B249, 361 (1985).

[9] M. Dine, L. Randall, and S. D. Thomas, Baryogenesis from flat directions of the supersymmetric standard model, Nucl. Phys. B458, 291 (1996).

[10] D. Bodeker and W. Buchmuller, Baryogenesis from the weak scale to the GUT scale, arXiv:2009.07294.

[11] P. A. R. Ade et al. (Planck Collaboration), Planck 2015 results. XIII. Cosmological parameters, Astron. Astrophys. 594, A13 (2016).

[12] A. Tan et al. (PandaX-II Collaboration), Dark Matter Results from First 98.7 Days of Data from the PandaX-II Experiment, Phys. Rev. Lett. 117, 121303 (2016).

[13] D. S. Akerib et al. (LUX Collaboration), Results from a Search for Dark Matter in the Complete LUX Exposure, Phys. Rev. Lett. 118, 021303 (2017).

[14] V. Khachatryan et al. (CMS Collaboration), Search for dark matter particles in proton-proton collisions at $\sqrt{s}=8 \mathrm{TeV}$ using the razor variables, J. High Energy Phys. 12 (2016) 088.

[15] G. Aad et al. (ATLAS Collaboration), Search for Dark Matter in Events with a Hadronically Decaying W or Z
Boson and Missing Transverse Momentum in $p p$ Collisions at $\sqrt{s}=8 \mathrm{TeV}$ with the ATLAS Detector, Phys. Rev. Lett. 112, 041802 (2014).

[16] D. Abercrombie et al., Dark matter benchmark models for early LHC Run-2 searches: Report of the ATLAS/CMS dark matter forum, Phys. Dark Universe 26, 100371 (2019).

[17] M. Aaboud et al. (ATLAS Collaboration), Search for dark matter at $\sqrt{s}=13 \mathrm{TeV}$ in final states containing an energetic photon and large missing transverse momentum with the ATLAS detector, Eur. Phys. J. C 77, 393 (2017).

[18] M. Aaboud et al. (ATLAS Collaboration), Search for Dark Matter Produced in Association with a Higgs Boson Decaying to $b \bar{b}$ Using $36 \mathrm{fb}^{-1}$ of $p p$ Collisions at $\sqrt{s}=$ $13 \mathrm{TeV}$ with the ATLAS Detector, Phys. Rev. Lett. 119, 181804 (2017).

[19] S. Nussinov, Technocosmology: Could a technibaryon excess provide a 'natural' missing mass candidate?, Phys. Lett. 165B, 55 (1985).

[20] D. B. Kaplan, A Single Explanation for Both the Baryon and Dark Matter Densities, Phys. Rev. Lett. 68, 741 (1992).

[21] S. M. Barr, R. S. Chivukula, and E. Farhi, Electroweak fermion number violation and the production of stable particles in the early universe, Phys. Lett. B 241, 387 (1990).

[22] S. M. Barr, Baryogenesis, sphalerons and the cogeneration of dark matter, Phys. Rev. D 44, 3062 (1991).

[23] S. Dodelson, B. R. Greene, and L. M. Widrow, Baryogenesis, dark matter and the width of the Z, Nucl. Phys. B372, 467 (1992).

[24] M. Fujii and T. Yanagida, A solution to the coincidence puzzle of Omega(B) and Omega (DM), Phys. Lett. B 542, 80 (2002).

[25] R. Kitano and I. Low, Dark matter from baryon asymmetry, Phys. Rev. D 71, 023510 (2005).

[26] G. R. Farrar and G. Zaharijas, Dark Matter and the Baryon Asymmetry, Phys. Rev. Lett. 96, 041302 (2006).

[27] R. Kitano, H. Murayama, and M. Ratz, Unified origin of baryons and dark matter, Phys. Lett. B 669, 145 (2008).

[28] S. B. Gudnason, C. Kouvaris, and F. Sannino, Towards working technicolor: Effective theories and dark matter, Phys. Rev. D 73, 115003 (2006).

[29] D. E. Kaplan, M. A. Luty, and K. M. Zurek, Asymmetric dark matter, Phys. Rev. D 79, 115016 (2009). 
[30] J. Shelton and K. M. Zurek, Darkogenesis: A baryon asymmetry from the dark matter sector, Phys. Rev. D 82, 123512 (2010).

[31] H. Davoudiasl, D. E. Morrissey, K. Sigurdson, and S. Tulin, Hylogenesis: A Unified Origin for Baryonic Visible Matter and Antibaryonic Dark Matter, Phys. Rev. Lett. 105, 211304 (2010).

[32] F. P. Huang and C. S. Li, Probing the baryogenesis and dark matter relaxed in phase transition by gravitational waves and colliders, Phys. Rev. D 96, 095028 (2017).

[33] M. R. Buckley and L. Randall, Xogenesis, J. High Energy Phys. 09 (2011) 009.

[34] T. Cohen, D. J. Phalen, A. Pierce, and K. M. Zurek, Asymmetric dark matter from a $\mathrm{GeV}$ hidden sector, Phys. Rev. D 82, 056001 (2010).

[35] M. T. Frandsen, S. Sarkar, and K. Schmidt-Hoberg, Light asymmetric dark matter from new strong dynamics, Phys. Rev. D 84, 051703(R) (2011).

[36] K. Petraki and R. R. Volkas, Review of asymmetric dark matter, Int. J. Mod. Phys. A 28, 1330028 (2013).

[37] K. M. Zurek, Asymmetric dark matter: Theories, signatures, and constraints, Phys. Rep. 537, 91 (2014).

[38] M. Ibe, A. Kamada, S. Kobayashi, and W. Nakano, Composite asymmetric dark matter with a dark photon portal, J. High Energy Phys. 11 (2018) 203.

[39] M. Ibe, A. Kamada, S. Kobayashi, T. Kuwahara, and W. Nakano, Ultraviolet completion of a composite asymmetric dark matter model with a dark photon portal, J. High Energy Phys. 03 (2019) 173.

[40] H. An, S. L. Chen, R. N. Mohapatra, and Y. Zhang, Leptogenesis as a common origin for matter and dark matter, J. High Energy Phys. 03 (2010) 124.

[41] A. Falkowski, J. T. Ruderman, and T. Volansky, Asymmetric dark matter from leptogenesis, J. High Energy Phys. 05 (2011) 106.

[42] D. S. M. Alves, S. R. Behbahani, P. Schuster, and J. G. Wacker, Composite inelastic dark matter, Phys. Lett. B 692, 323 (2010).

[43] D. S. M. Alves, S. R. Behbahani, P. Schuster, and J. G. Wacker, The cosmology of composite inelastic dark matter, J. High Energy Phys. 06 (2010) 113.

[44] M. Blennow, E. Fernandez-Martinez, O. Mena, J. Redondo, and P. Serra, Asymmetric dark matter and dark radiation, J. Cosmol. Astropart. Phys. 07 (2012) 022.

[45] Y. Bai and P. Schwaller, Scale of dark QCD, Phys. Rev. D 89, 063522 (2014).

[46] V. Beylin, M. Khlopov, V. Kuksa, and N. Volchanskiy, New physics of strong interaction and dark universe, Universe 6 , 196 (2020).

[47] M. Y. Khlopov, G. M. Beskin, N. E. Bochkarev, L. A. Pustylnik, and S. A. Pustylnik, Observational physics of mirror World, Sov. Astron. 35, 21 (1991).

[48] S. I. Blinnikov and M. Khlopov, Possible astronomical effects of mirror particles, Sov. Astron. 27, 371 (1983).

[49] S. I. Blinnikov and M. Y. Khlopov, On possible effects of 'mirror' particles, Sov. J. Nucl. Phys. 36, 472 (1982).

[50] M. J. Strassler and K. M. Zurek, Echoes of a hidden valley at hadron colliders, Phys. Lett. B 651, 374 (2007).
[51] M. J. Strassler and K. M. Zurek, Discovering the Higgs through highly-displaced vertices, Phys. Lett. B 661, 263 (2008).

[52] M. J. Strassler, Possible effects of a hidden valley on supersymmetric phenomenology, arXiv:hep-ph/0607160.

[53] T. Han, Z. Si, K. M. Zurek, and M. J. Strassler, Phenomenology of hidden valleys at hadron colliders, J. High Energy Phys. 07 (2008) 008.

[54] M. Verducci (ATLAS Collaboration), Hidden Valley search at ATLAS, J. Phys. Conf. Ser. 335, 012068 (2011).

[55] Y. F. Chan, M. Low, D. E. Morrissey, and A. P. Spray, LHC signatures of a minimal supersymmetric hidden valley, J. High Energy Phys. 05 (2012) 155.

[56] M. J. Strassler, On the phenomenology of hidden valleys with heavy flavor, arXiv:0806.2385.

[57] T. Cohen, M. Lisanti, and H. K. Lou, Semivisible Jets: Dark Matter Undercover at the LHC, Phys. Rev. Lett. 115, 171804 (2015).

[58] P. Schwaller, D. Stolarski, and A. Weiler, Emerging jets, J. High Energy Phys. 05 (2015) 059.

[59] H. Beauchesne, E. Bertuzzo, G. Grilli Di Cortona, and Z. Tabrizi, Collider phenomenology of Hidden Valley mediators of spin 0 or $1 / 2$ with semivisible jets, J. High Energy Phys. 08 (2018) 030.

[60] A. Pierce, B. Shakya, Y. Tsai, and Y. Zhao, Searching for confining hidden valleys at LHCb, ATLAS, and CMS, Phys. Rev. D 97, 095033 (2018).

[61] H. Beauchesne, E. Bertuzzo, and G. Grilli Di Cortona, Dark matter in Hidden Valley models with stable and unstable light dark mesons, J. High Energy Phys. 04 (2019) 118.

[62] S. Renner and P. Schwaller, A flavoured dark sector, J. High Energy Phys. 08 (2018) 052.

[63] M. Park and M. Zhang, Tagging a jet from a dark sector with jet-substructures at colliders, Phys. Rev. D 100, 115009 (2019).

[64] H. Mies, C. Scherb, and P. Schwaller, Collider constraints on dark mediators, J. High Energy Phys. 04 (2021) 049.

[65] D. Kar and S. Sinha, Exploring jet substructure in semivisible jets, SciPost Phys. 10, 084 (2021).

[66] E. Bernreuther, T. Finke, F. Kahlhoefer, M. Krämer, and A. Mück, Casting a graph net to catch dark showers, SciPost Phys. 10, 046 (2021).

[67] T. Cohen, J. Doss, and M. Freytsis, Jet substructure from dark sector showers, J. High Energy Phys. 09 (2020) 118.

[68] S. H. Lim and M. M. Nojiri, Morphology for jet classification, arXiv:2010.13469.

[69] S. Knapen, J. Shelton, and D. Xu, Perturbative benchmark models for a dark shower search program, Phys. Rev. D 103, 115013 (2021).

[70] D. Linthorne and D. Stolarski, Triggering on emerging jets, arXiv:2103.08620.

[71] H. Beauchesne and G. Grilli di Cortona, Classification of dark pion multiplets as dark matter candidates and collider phenomenology, J. High Energy Phys. 02 (2020) 196.

[72] J. B. Guimarães da Costa et al. (CEPC Study Group), CEPC conceptual design report: Volume 2-Physics \& detector, arXiv:1811.10545.

[73] W. Buchmuller, P. Di Bari, and M. Plumacher, Leptogenesis for pedestrians, Ann. Phys. (Amsterdam) 315, 305 (2005). 
[74] S. Davidson, E. Nardi, and Y. Nir, Leptogenesis, Phys. Rep. 466, 105 (2008).

[75] L. Covi, E. Roulet, and F. Vissani, $C P$ violating decays in leptogenesis scenarios, Phys. Lett. B 384, 169 (1996).

[76] M. D’Onofrio, K. Rummukainen, and A. Tranberg, Sphaleron Rate in the Minimal Standard Model, Phys. Rev. Lett. 113, 141602 (2014).

[77] J. A. Harvey and M. S. Turner, Cosmological baryon and lepton number in the presence of electroweak fermion number violation, Phys. Rev. D 42, 3344 (1990).

[78] K. Enqvist, S. Nadathur, T. Sekiguchi, and T. Takahashi, Constraints on decaying dark matter from weak lensing and cluster counts, J. Cosmol. Astropart. Phys. 04 (2020) 015.

[79] T. Lin, Dark matter models and direct detection, Proc. Sci., 333 (2019) 009 [arXiv:1904.07915].

[80] R. Essig, T. Volansky, and T. T. Yu, New constraints and prospects for sub-GeV dark matter scattering off electrons in xenon, Phys. Rev. D 96, 043017 (2017).

[81] E. Aprile et al. (XENON Collaboration), Low-mass dark matter search using ionization signals in XENON100, Phys. Rev. D 94, 092001 (2016); Erratum, Phys. Rev. D 95, 059901 (2017).

[82] P. Agnes et al. (DarkSide Collaboration), Constraints on Sub-GeV Dark-Matter-Electron Scattering from the DarkSide-50 Experiment, Phys. Rev. Lett. 121, 111303 (2018).

[83] A. Alloul, N. D. Christensen, C. Degrande, C. Duhr, and B. Fuks, FeynRules 2.0-A complete toolbox for tree-level phenomenology, Comput. Phys. Commun. 185, 2250 (2014).

[84] C. Degrande, C. Duhr, B. Fuks, D. Grellscheid, O. Mattelaer, and T. Reiter, UFO-The Universal FeynRules Output, Comput. Phys. Commun. 183, 1201 (2012).

[85] J. Alwall, M. Herquet, F. Maltoni, O. Mattelaer, and T. Stelzer, MadGraph5: Going beyond, J. High Energy Phys. 06 (2011) 128.

[86] T. Sjöstrand, S. Ask, J. R. Christiansen, R. Corke, N. Desai, P. Ilten, S. Mrenna, S. Prestel, C. O. Rasmussen, and P. Z. Skands, An introduction to PYTHIA 8.2, Comput. Phys. Commun. 191, 159 (2015).
[87] J. de Favereau, C. Delaere, P. Demin, A. Giammanco, V. Lemaître, A. Mertens, and M. Selvaggi (DELPHES 3 Collaboration), DELPHES 3, A modular framework for fast simulation of a generic collider experiment, J. High Energy Phys. 02 (2014) 057.

[88] M. Cacciari, G. P. Salam, and G. Soyez, The anti-k(t) jet clustering algorithm, J. High Energy Phys. 04 (2008) 063.

[89] M. Cacciari, G. P. Salam, and G. Soyez, FastJet user manual, Eur. Phys. J. C 72, 1896 (2012).

[90] ATLAS Collaboration, Search for long-lived neutral particles decaying into displaced lepton jets in proton-proton collisions at $\sqrt{s}=13 \mathrm{TeV}$ with the ATLAS detector, Report No. ATLAS-CONF-2016-042.

[91] J. Fiaschi and M. Klasen, Slepton pair production at the LHC in NLO + NLL with resummation-improved parton densities, J. High Energy Phys. 03 (2018) 094.

[92] H. Baer, T. Barklow, K. Fujii, Y. Gao, A. Hoang, S. Kanemura, J. List, H. E. Logan, A. Nomerotski, M. Perelstein et al., The International Linear Collider Technical Design Report_-Volume 2: Physics, arXiv:1306.6352.

[93] H. Abramowicz et al. (CLIC Detector and Physics Study), Physics at the CLIC e+e- linear collider-Input to the Snowmass process 2013, arXiv:1307.5288.

[94] M. Bicer et al. (TLEP Design Study Working Group), First look at the physics case of TLEP, J. High Energy Phys. 01 (2014) 164.

[95] A. Falkowski, J. T. Ruderman, T. Volansky, and J. Zupan, Hidden Higgs decaying to lepton jets, J. High Energy Phys. 05 (2010) 077.

[96] A. Falkowski, J. T. Ruderman, T. Volansky, and J. Zupan, Discovering Higgs Decays to Lepton Jets at Hadron Colliders, Phys. Rev. Lett. 105, 241801 (2010).

[97] H. Liang and M. Ruan, Detector performance and physics potential at CEPC, Int. J. Mod. Phys. Conf. Ser. 46, 1860086 (2018).

[98] Private discussion with CEPC group members. 